\section{6/\#233 CLINICAL AND MOLECULAR CHARACTERISTICS OF ARIEL3 PATIENTS WHO DERIVED EXCEPTIONAL BENEFIT FROM RUCAPARIB MAINTENANCE TREATMENT FOR HIGH-GRADE OVARIAN CANCER (HGOC)}

\begin{abstract}
1,2RL Coleman ${ }^{*},{ }^{3} \mathrm{~A}$ Oza, ${ }^{4,5} \mathrm{D}$ Lorusso, ${ }^{6} \mathrm{C}$ Aghajanian, ${ }^{7} \mathrm{~A}$ Oaknin, ${ }^{8} \mathrm{~A}$ Dean, ${ }^{9} \mathrm{~N}$ Colombo,
\end{abstract} ${ }^{10} \mathrm{~J}$ Weberpals, ${ }^{11} \mathrm{~A}$ Clamp, ${ }^{5} \mathrm{G}$ Scambia, ${ }^{12} \mathrm{~A}$ Leary, ${ }^{13} \mathrm{R}$ Holloway, ${ }^{14} \mathrm{M}$ Amenedo Gancedo, ${ }^{15} \mathrm{P}$ Fong, ${ }^{16} \mathrm{~J}$ Goh, ${ }^{17} \mathrm{D}$ O'Malley, ${ }^{18} \mathrm{~L}$ Maloney, ${ }^{19} \mathrm{~S}$ Goble, ${ }^{20} \mathrm{~T}$ Kwan, ${ }^{21} \mathrm{~J}$ Ledermann. ${ }^{1}$ University of Texas MD anderson Cancer Center, Department of Gynecologic Oncology and Reproductive Medicine, Houston, USA; ${ }^{2}$ US Oncology Research, Research, The Woodlands, USA; ${ }^{3}$ Princess Margaret Cancer Centre, University Health Network, Division of Medical Oncology and Hematology, Toronto, Canada; ${ }^{4}$ Fondazione IRCCS, Istituto Nazionale dei Tumori, Multicenter Italian Trials In Ovarian Cancer and Gynecologic Malignancies and Gynecologic Oncology Unit, Milan, Italy; ${ }^{5}$ Fondazione Policlinico Universitario A. Gemelli IRCCS and Scientific Directorate, Gynecologic Oncology Unit, Rome, Italy; ${ }^{6}$ Memorial Sloan Kettering Cancer Center, Department of Medicine, New York, USA; ${ }^{7}$ Vall d'Hebron Institute of Oncology (VHIO), Hospital Universitari Vall d'Hebron, Vall d'Hebron Barcelona Hospital Campus, Gynecologic Cancer Program, Barcelona, Spain; ${ }^{8}$ St John of God Subiaco Hospital, Department of Oncology, Subiaco, Australia; ${ }^{9}$ University of Milan-Bicocca and European Institute of Oncology (IEO) IRCCS, Gynecologic Cancer Program, Milan, Italy; ${ }^{10}$ Ottawa Hospital Research Institute, Division of Gynecologic Oncology, Ottawa, Canada; ${ }^{11}$ The Christie NHS Foundation Trust and University of Manchester, Department of Medical Oncology, Manchester, UK; ${ }^{12}$ Gustave Roussy Cancer Center, INSERM U981, and Groupe d'Investigateurs Nationaux pour I'Etude des Cancers Ovariens (GINECO), Gynecological Unit, Villejuif, France; ${ }^{13}$ Florida Hospital Cancer Institute, Department of Gynecologic Oncology, Orlando, USA; ${ }^{14}$ Oncology Center of Galicia, Medical Oncology Department, La Coruña, Spain; ${ }^{15}$ Auckland City Hospital and University of Auckland, Medical Oncology Department, Auckland, New Zealand; ${ }^{16}$ Royal Brisbane and Women's Hospital, Herston, and University of Queensland, Department of Oncology, Cancer Care Services, St Lucia, Australia; ${ }^{17}$ The Ohio State University, James Cancer Center, Division of Gynecologic Oncology, Columbus, USA; ${ }^{18}$ Clovis Oncology, Inc., Clinical Development, Boulder, USA; ${ }^{19}$ Clovis Oncology, Inc., Biostatistics, Boulder, USA; ${ }^{20}$ Clovis Oncology, Inc., Molecular Diagnostics and Translational Medicine, Boulder, USA; ${ }^{21}$ UCL Cancer Institute, University College London and UCL Hospitals, Department of Oncology, London, UK

\subsection{6/ijgc-2021-IGCS.16}

Objectives ARIEL3 is a placebo-controlled randomized trial of the PARP inhibitor (PARPi) rucaparib as maintenance treatment in HGOC patients who responded to the latest line of platinum therapy (NCT01968213). Rucaparib improved progression-free survival (PFS) across all predefined subgroups. Here, we present an exploratory analysis of characteristics associated with exceptional benefit from rucaparib.

Methods Patients were randomized 2:1 to rucaparib $600 \mathrm{mg}$ BID or placebo. As of 31 Dec 2019 (data cutoff), 33/375 $(9 \%)$ and $1 / 189(0.5 \%)$ patients were still ongoing and receiving rucaparib or placebo. Molecular features (genomic alterations, BRCA1 promoter methylation) and baseline clinical

\begin{tabular}{|c|c|c|c|c|c|c|}
\hline \multirow[b]{2}{*}{ Baseline characteristics } & \multicolumn{3}{|c|}{ Rucaparib (N=375) } & \multicolumn{3}{|c|}{ Placebo (N=189) } \\
\hline & $\begin{array}{c}\text { Exceptional } \\
\text { benefit } \\
n=79\end{array}$ & $\begin{array}{c}\text { Short-term } \\
\text { subgroup } \\
n=64\end{array}$ & $\begin{array}{l}\text { Odds ratio } \\
\text { (95\% Cl) }\end{array}$ & $\begin{array}{c}\text { Exceptional } \\
\text { benefit } \\
n=4\end{array}$ & $\begin{array}{l}\text { Short-term } \\
\text { subgroup } \\
n=62\end{array}$ & $\begin{array}{l}\text { Odds ratio } \\
\text { (95\% Cl) }\end{array}$ \\
\hline No measurable disease & $58(73 \%)$ & $26(416)$ & $\begin{array}{c}4.0 \\
(20-8.0)^{p}\end{array}$ & $3(75 \%)$ & $33(53 \%)$ & $\begin{array}{c}2.6 \\
(0.4-35.3)\end{array}$ \\
\hline $\begin{array}{r}\text { Complete response to latest } \\
\text { platinum }\end{array}$ & $31(396)$ & $13(20 \%)$ & $\begin{array}{c}2.5 \\
(122-5.3)^{\circ}\end{array}$ & 1 (25\%) & $11(18 \%)$ & $\begin{array}{c}1.5 \\
(0.1-11.2) \\
\end{array}$ \\
\hline $\begin{array}{r}\begin{array}{r}\text { Penultimate platinum-free } \\
\text { interval }>12 \mathrm{mo}\end{array}\end{array}$ & $55(7086)$ & $30(47 \times)$ & $\begin{array}{c}2.6 \\
(13-5.2)^{\circ}\end{array}$ & $4(100 \%)$ & $29(47 \times 6)$ & NA \\
\hline BRCA mutation & $46(588)$ & $12(1966)$ & $\begin{array}{c}6.0 \\
(2.8-13.3)^{\circ}\end{array}$ & $3(75 \%)$ & $25(4036)$ & $\begin{array}{c}4.4 \\
(0.6-59.0)\end{array}$ \\
\hline BRCAwt/LOH-high & $18(236)$ & $19(30 x)$ & $\begin{array}{c}0.7 \\
(0.3-1.5)\end{array}$ & 0 & $14(236)$ & NA \\
\hline BRCAwat/LOH-low & $8(10 \%)$ & $28(44 \times)$ & $\begin{array}{c}0.14 \\
(0.1-0.4)^{2}\end{array}$ & $1(25 \%)$ & $16(26 \%)$ & $\begin{array}{c}1.0 \\
(0.1-6.8)\end{array}$ \\
\hline $\begin{array}{l}\text { BRCAvit + RAD51C/D } \\
\text { mutation }^{b}\end{array}$ & $6(8 \%)$ & 0 & NA & 0 & 0 & NA \\
\hline $\begin{array}{r}\text { BRCAwrt + high BRCA1 } \\
\text { promoter methylation }\end{array}$ & $6 / 25(24 \%)$ & $7 / 47(15 \%)$ & $\begin{array}{c}1.8 \\
(0.5-6.0)\end{array}$ & $0 / 1$ & $5 / 29(17 \%)$ & NA \\
\hline
\end{tabular}

BRCAwt, BRCA wild type; LOH, loss of heterozygosity; NA, not applicable ${ }^{\mathrm{a}}$ Significant result; ${ }^{\mathrm{b}} \mathrm{P}=0.033$ (Fisher's exact test); cln samples with known methylation status characteristics were compared between patients who derived exceptional benefit (PFS $\geq 2$ yrs), and those with disease progression on first scan $(\approx 12$ wks; the short-term subgroup) within each treatment arm.

Results of 564 patients, 79/375 (21\%) in the rucaparib arm and $4 / 189(2 \%)$ in the placebo arm showed exceptional benefit. Within the rucaparib arm, exceptional benefit patients had more favorable clinical prognostic factors at baseline versus the short-term subgroup (Table). Although BRCA mutations were enriched in the rucaparib exceptional benefit subgroup, $33 / 79(42 \%)$ of these patients were BRCA wild type. Patterns of enrichment varied among other biomarkers. Overall trends were similar in the placebo arm.

Conclusions Exceptional benefit in ARIEL3 was more common in, but not exclusive to, patients with favorable clinical characteristics and known mechanisms of PARPi sensitivity. Our results suggest rucaparib can deliver exceptional benefit to a diverse set of patients with HGOC.

\section{7/\#15 EFFICACY AND SAFETY OF NIRAPARIB MAINTENANCE TREATMENT IN PLATINUM- SENSITIVE RECURRENT OVARIAN CANCER AFTER SHORTER OR LONGER CHEMOTHERAPY: A POST HOC SUBGROUP ANALYSIS}

${ }^{1} \mathrm{Y}$ Gao*, ${ }^{2} \mathrm{X}$ Wu, ${ }^{3} \mathrm{~J} Z \mathrm{Zhu},{ }^{4} \mathrm{R}$ Yin, ${ }^{5} \mathrm{~J}$ Yang, ${ }^{6} \mathrm{~J} \mathrm{Liu,}{ }^{7} \mathrm{~J}$ Wang, ${ }^{8} \mathrm{~L} \mathrm{Wu},{ }^{9} \mathrm{Z} \mathrm{Liu},{ }^{10} \mathrm{D}$ Wang, ${ }^{11} \mathrm{G}$ Lou, ${ }^{12} \mathrm{H}$ Yang, ${ }^{13} \mathrm{Q}$ Zhou, ${ }^{14} \mathrm{~B}$ Kong, ${ }^{15} \mathrm{Y}$ Huang, ${ }^{16} \mathrm{~L}$ Chen, ${ }^{17} \mathrm{G} \mathrm{Li},{ }^{18} \mathrm{R}$ An, ${ }^{19} \mathrm{~T}$ Tan, ${ }^{19} \mathrm{~J}$ Dong. ${ }^{1}$ Peking University Cancer Hospital and Institute, Department of Gynecology, Beijing, China; ${ }^{2}$ Fudan University Shanghai Cancer Center, Department of Gynecologic Oncology, Shanghai, China; ${ }^{3}$ Cancer Hospital of the University of Chinese Academy of Sciences (Zhejiang Cancer Hospital), Department of Gynecologic Oncology, Hangzhou, China; ${ }^{4}$ West China Second University Hospital, Sichuan University, Department of Obstetrics and Gynecology; Key Laboratory of Birth Defects and Related Diseases of Women and Children (sichuan University), Ministry of Education, Chengdu, China; ${ }^{5}$ Peking Union Medical College Hospital, Chinese Academy of Medical Sciences and Peking Union Medical College, Department of Gynecologic Oncology, Beijing, China; ${ }^{6}$ Sun Yat-sen University Cancer Center, Department of Gynecologic Oncology, Guangzhou, China; ${ }^{7}$ Hunan Cancer Hospital, the Affiliated Cancer Hospital of Xiangya School of Medicine, Central South University, Department of Gynecologic Oncology, Changsha, China; ${ }^{8}$ National Cancer Center/Cancer Hospital, Chinese Academy of Medical Sciences and Peking Union Medical College, Department of Gynecologic Oncology, Beijing, China; ${ }^{9}$ The First Hospital of Jilin University, Department of Oncology, Changchun, China; ${ }^{10}$ Cancer Hospital of China Medical University, Liaoning Cancer Hospital and Institute, Department of Gynecology, Shenyang, China; ${ }^{11}$ Harbin Medical University Cancer Hospital, Department of Gynecology, Harbin, China; ${ }^{12}$ The Third Affiliated Hospital of Kunming Medical University/Yunnan Cancer Hospital, Department of Gynecologic Oncology, Kunming, China; ${ }^{13}$ Chongqing University Cancer Hospital, Gynecological Oncology Center, Chongqing, China; ${ }^{14}$ Qilu Hospital of Shandong University, Department of Obstetrics and Gynecology, Jinan, China; ${ }^{15} \mathrm{Hubei}$ Cancer Hospital, Department of Gynecologic Oncology, Wuhan, China; ${ }^{16}$ Affiliated Cancer Hospital of Guangzhou Medical University, Department of Gynecological Oncology, Guangzhou, China; ${ }^{17}$ Union Hospital, Tongji Medical College, Huazhong University of Science and Technology, Cancer Center, Wuhan, China; ${ }^{18}$ The First Affiliated Hospital of Xi'an Jiaotong University, Department of Obstetrics and Gynecology, Xi'an, China; ${ }^{19}$ Zai Lab (Shanghai) Co., Ltd, Randd Department, Shanghai, China

\subsection{6/ijgc-2021-IGCS.17}

Objectives Traditionally $\geq 6$ cycles of platinum-containing chemotherapy (Pt-chemo) are recommended for platinum-sensitive recurrent ovarian cancer (PSROC). PARP inhibitor maintenance treatment (MT) can be initiated upon clinical complete/ partial response (CR/PR) after $\geq 4$ cycles of chemotherapy. Shorter chemotherapy may improve patient experience without compromising efficacy. This study aims to compare the efficacy and safety of niraparib to placebo as MT administered after $\leq 4$ or $>4$ cycles of Pt-chemo. 
Methods This is a post hoc analysis of the published NORA phase III study (NCT03705156). Adults with PSROC and CR/ $\mathrm{PR}$ to most recent Pt-chemo were randomized 2:1 to niraparib or placebo. Primary endpoint was PFS by BICR. Subgroups comprised patients with $\leq 4$ or $>4$ cycles of most recent Pt-chemo.

Results Table 1 summarizes key baseline characteristics which were overall balanced between groups. Median (95\% CI) PFS was 18.37 months (8.54-not estimable [NE], $\leq 4$-cycle/niraparib) versus 3.88 months (3.68-7.43, $\leq 4$-cycle/placebo; $\mathrm{HR}=0.36[\mathrm{p}=0.0016])$, and was 18.33 months $(10.28-\mathrm{NE}$, $>4$-cycle/niraparib) versus 5.49 months (3.71-5.75, >4-cycle/ placebo; $\mathrm{HR}=0.33[\mathrm{p}<0.0001]$ ) (figure 1 ). Overall safety profiles were comparable between $\leq 4$-cycle/niraparib and $>4$ cycle/niraparib, with similar percentages of patients experiencing neutrophil count decrease $(60.4 \%$; 58.1\%), anemia

Abstract 0017/\#15 Table 1 Key baseline characteristics for subgroups with $\leq 4$ or $>4$ treatment cycles of most recent PT-chemo

\begin{tabular}{|c|c|c|c|c|}
\hline \multirow{2}{*}{$n(x)$} & \multicolumn{2}{|c|}{ Niraparib } & \multicolumn{2}{|c|}{ Placebo } \\
\hline & $\begin{array}{l}\leq 4 \text { cycles } \\
\text { Pt-Chemo }\end{array}$ & $\begin{array}{l}>4 \text { cycles } \\
\text { Pt-Chemo }\end{array}$ & $\begin{array}{l}\leq 4 \text { cycles } \\
\text { Pt-Chemo }\end{array}$ & $\begin{array}{l}>4 \text { cycles } \\
\text { Pt-Chemo }\end{array}$ \\
\hline \multicolumn{5}{|c|}{ Best response to most recent Pt-chemo $0^{a}$} \\
\hline$C R$ & $28(58.3)$ & $58(45.0)$ & $18(60.0)$ & $29(50.0)$ \\
\hline PR & $20(41.7)$ & $70(54.3)$ & $12(40.0)$ & $29(50.0)$ \\
\hline \multicolumn{5}{|l|}{ BRCA mutation status } \\
\hline Germline BRCA mutation & $17(35.4)$ & $48(37.2)$ & $10(33.3)$ & $25(43.1)$ \\
\hline Non-germline $B R C A$ mutation & $31(64.6)$ & $81(62.8)$ & $20(66.7)$ & $33(56.9)$ \\
\hline \multicolumn{5}{|c|}{ Platinum sensitivity after penultimate Pt-chemo } \\
\hline Complete & $29(60.4)$ & $92(71.3)$ & $18(60.0)$ & $42(72.4)$ \\
\hline Partial & $19(396)$ & $37(287)$ & $12(400)$ & $16(27.6)$ \\
\hline
\end{tabular}

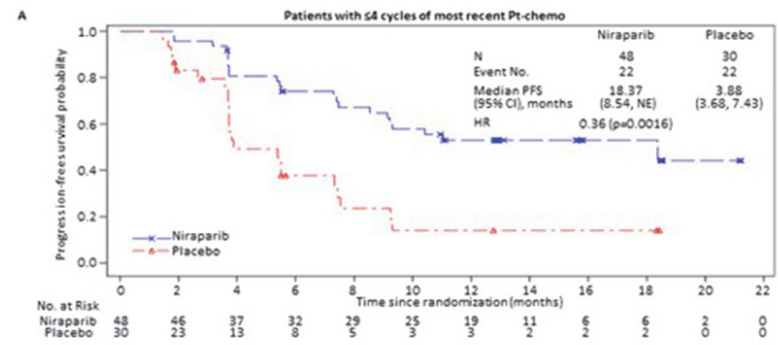

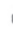
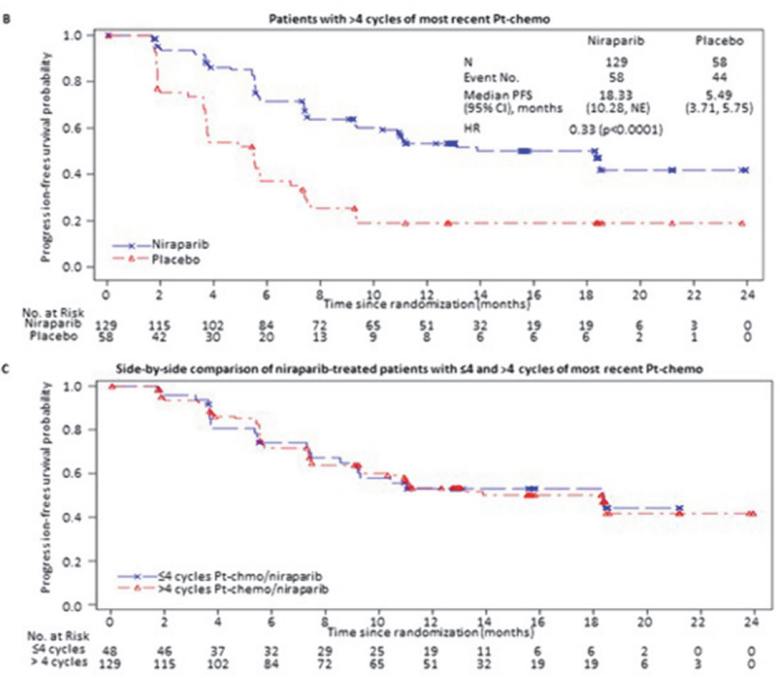

Abstract 0017/\#15 Figure 1 PFS by blinded independent central review (interaction-to-treat)
(50.0\%; 55.0\%), and platelet count decrease (45.8\%; 58.1\%). Composition of grade $\geq 3$ TEAEs was consistent with the overall NORA results.

Conclusions Similar niraparib-versus-placebo PFS benefits were observed after $\leq 4$-cycle or $>4$-cycle Pt-chemo in CR/PR patients. The efficacy and safety of niraparib MT after shorter Pt-chemo remain to be verified in larger samples.

\section{Oral Featured Posters}

\section{OP001/\#162 SENTINEL LYMPH NODE MAPPING IN EARLY- STAGE CERVICAL CANCER - A NATIONAL PROSPECTIVE MULTICENTER STUDY (SENTIREC TRIAL)}

1,2S Sponholtz*, ${ }^{3,4} \mathrm{O}$ Mogensen, ${ }^{2,5,6} \mathrm{M}$ Hildebrandt, ${ }^{2,7} \mathrm{D}$ Schledermann, ${ }^{8} \mathrm{E}$ Parner, ${ }^{1} \mathrm{~A}$ Markauskas, ${ }^{9} \mathrm{~L}$ Frøding, ${ }^{3} \mathrm{~K}$ Fuglsang, ${ }^{5} \mathrm{M}$ Vilstrup, ${ }^{3,4} \mathrm{~S}$ Bjørnholt, ${ }^{2,3,4} \mathrm{P}$ Jensen. ${ }^{1}$ Odense University Hospital, Department of Gynecology and Obstetrics, Odense C, Denmark; ${ }^{2}$ University of Southern Denmark, Department of Clinical Research, Odense C, Denmark; ${ }^{3}$ Aarhus University Hospital, Department of Gynecology and Obstetrics, Aarhus N, Denmark; ${ }^{4}$ Aarhus University, Institute of Clinical Medicine, Aarhus, Denmark; ${ }^{5}$ Odense University Hospital, Department of Nuclear Medicine, Odense C, Denmark; ${ }^{6}$ Odense University Hospital and University of Southern Denmark, Center For Innovative Medical Technology, Odense C, Denmark; ${ }^{7}$ Odense University Hospital, Department of Pathology, Odense C, Denmark;

${ }^{8}$ Aarhus University, Department of Public Health, Aarhus, Denmark; ${ }^{9}$ Copenhagen University Hospital, Department of Gynecology, Copenhagen, Denmark

\subsection{6/ijgc-2021-IGCS. 18}

Objectives Sentinel lymph node (SLN) mapping may replace staging radical pelvic lymphadenectomy in women with earlystage cervical cancer. In a national multicenter setting, we evaluated SLN mapping in women with early-stage cervical cancer and investigated the accuracy of SLN mapping and FDG-PET/CT in tumors $>20 \mathrm{~mm}$.

Methods We prospectively included women with early-stage cervical cancer from March 2017-January 2021 to undergo SLN mapping. Women with tumors $>20 \mathrm{~mm}$ underwent completion pelvic lymphadenectomy and removal of FDG-PET/CT positive nodes. We determined SLN detection rates, incidence of nodal disease, sensitivity and negative predictive value (NPV) of SLN mapping, and the sensitivity, specificity, NPV, and positive predictive value (PPV) of FDG-PET/CT.

Results We included 245 women, and 38 (15.5\%) had nodal metastasis. The SLN detection rate was 96.3\% (236/245), with $82.0 \%(201 / 245)$ bilateral detection. In a stratified analysis of 103 women with tumors $>20 \mathrm{~mm}, 27$ (26.2\%) had nodal metastases. The sensitivity of SLN mapping adhering to the algorithm was 96.3\% (95\% CI 81.0-99.9\%) and the NPV 98.7\% (95\% CI 93.0-100\%). For FDG-PET/ CT imaging the sensitivity was $14.8 \%$ (95\% CI 4.2-33.7\%), the specificity $85.5 \% \quad(95 \%$ CI $75.6-92.5 \%)$, the NPV 73.9\% (95\% CI 63.4-82.7\%), and the PPV 26.7\% (95\% CI 7.8-55.1\%).

Conclusions Our results suggest that SLN mapping is a reliable method in women with early-stage cervical cancer. However, until the oncological safety is established, we recommend completion pelvic lymphadenectomy in women with tumors $>20$ mm. FDG-PET/CT seems redundant for nodal staging in women with early-stage cervical cancer. 